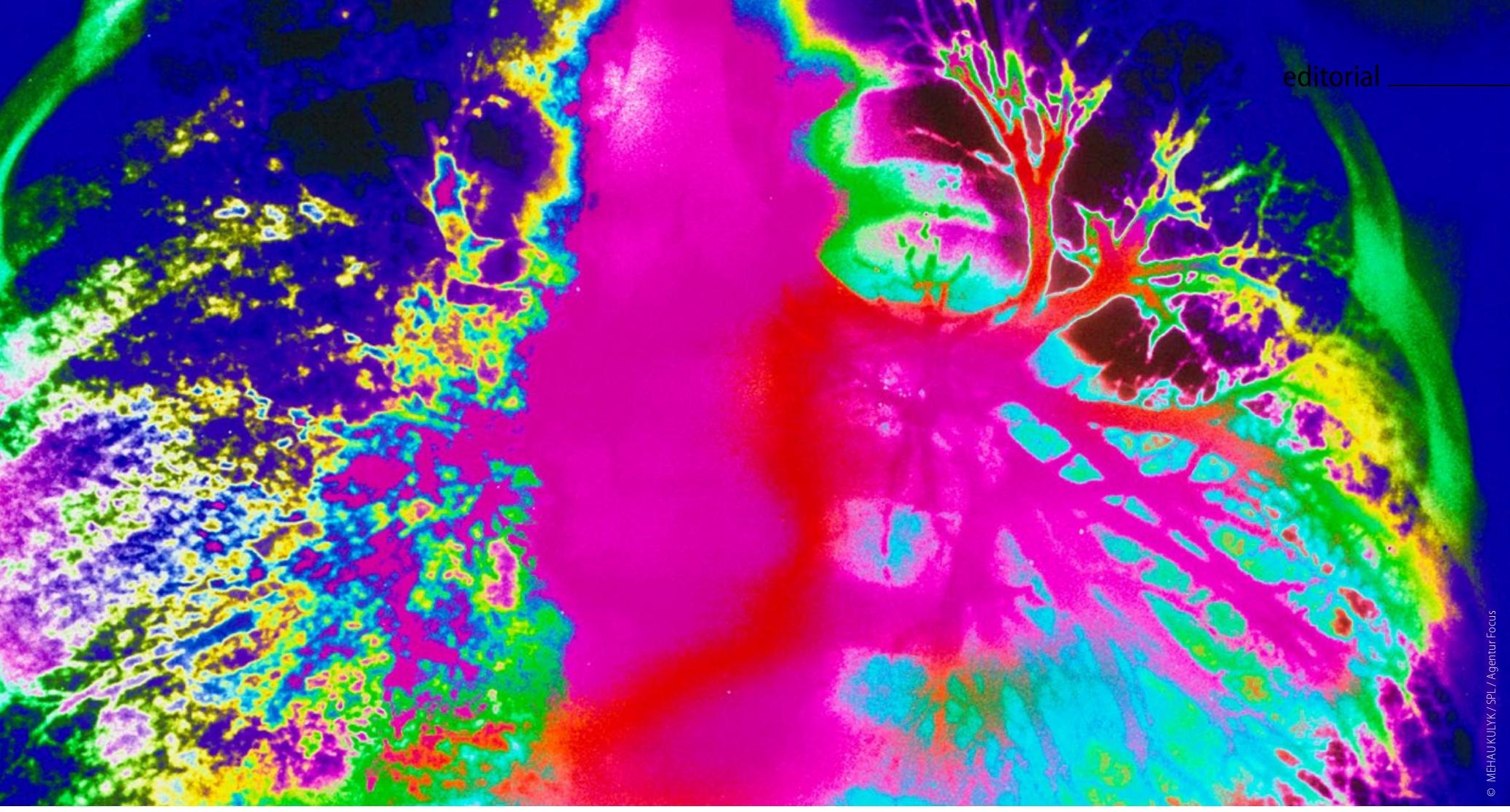

\title{
Mehr Aufmerksamkeit und Hilfe für Betroffene
}

Bronchiektasen, die nicht mit einer Mukoviszidose (cystische Fibrose, CF) assoziiert sind (Non-CFBronchiektasen), waren lange Zeit eine vernachlässigte Erkrankung. Bis heute existieren nur wenige kontrollierte Studien, sodass kaum evidenzbasierte Therapieempfehlungen abgegeben werden können. Mit dem seit Juni 2015 rekrutierenden deutschen Bronchiektasen-Register PROGNOSIS sollen zukünftig unter anderem wichtige epidemiologische Fragen beantwortet werden.

In Europa werden Bronchiektasen mehr und mehr zum „Hot Topic“ der Pneumologie. Und auch in Deutschland bekommt diese Lungenerkrankung langsam mehr Aufmerksamkeit. Non-CF-Bronchiektasen sind eine ätiologisch heterogene Erkrankung und dementsprechend häufig eine diagnostische und therapeutische Herausforderung. Charakteristisch ist eine weitgehend einheitliche Erkrankungsmanifestation mit permanenter Erweiterung der Bronchien und Bronchiolen sowie ein Circulus vitiosus aus chronischer Infektion, Inflammation, Sekretverhalt und der Zerstörung bronchialer Strukturen [1]. Folglich kommt der Diagnose der zugrundeliegenden Krankheit eine zentrale Bedeutung hinsichtlich einer zielgerichteten Therapie zu [2].

Die weiteren Ziele der Behandlung von Patienten mit Bronchiektasen orientieren sich am Pathomechanismus und sind die Verbesserung der Sekret-Clearance bzw. -Drainage, die stadiengerechte Therapie der Atemwegsobstruktion, die Therapie der chronischen Infektion und der zum Progress führenden chronischen Inflammation sowie die Prävention und das Management bei akuten Exazerbationen [3].

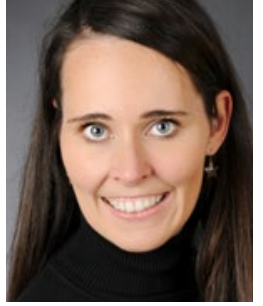

Dr. med. Jessica Rademacher

Medizinische Hochschule Hannover (MHH) KLINIK FÜR PNEUMOLOGIE

Carl-Neuberg-Str. 1

30625 Hannover

Rademacher.Jessica@mh-hannover.de

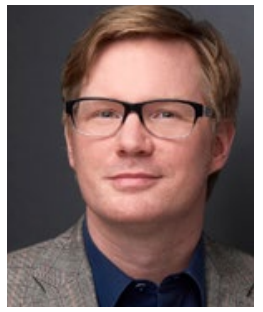

Dr. med. Felix C. Ringshausen

Medizinische Hochschule Hannover (MHH) KLINIK FÜR PNEUMOLOGIE

Carl-Neuberg-Str. 1

30625 Hannover

Ringshausen.Felix@mh-hannover.de 


\section{Hier steht eine Anzeige.}

Springer 


\section{Kenntnis der Ätiologie ist essenziell}

Die Diagnostik von Bronchiektasen ist häufig sehr kostenintensiv und nur in wenigen Zentren durchführbar. Sie kann jedoch in bis zu zwei Dritteln der Fälle die Ursache klären, die dann wiederum bei etwa einem Drittel der Patienten von besonderer Bedeutung für das weitere Management ist (zum Beispiel beim Immundefekt) [4]. Patienten mit Bronchiektasen werden meist ambulant und bei niedergelassenen Pneumologen behandelt [5]. Die aufwendige Diagnostik einschließlich des Ausschlusses einer CF (mittels Schweißtest, Genetik und/oder weiterführender

\section{"This is the age of bronchiectasis"}

(John R. Hurst in Lancet Respiratory Medicine, 2014)

elektrophysiologischer Untersuchungen der CFTR-Funktion, zum Beispiel durch die nasale Potenzialdifferenzmessung) und einer Primären Ciliären Dyskinesie (PCD; mittels nasalem exhalativen NO, Hochfrequenz-videomikroskopischer Analyse einer Nasenbürstenzytologie, Elektronenmikroskopie, Immunfluoreszenz und Genetik) sind nicht flächendeckend verfügbar.

Ein Modell für die Zukunft könnten über Deutschland verteilte spezialisierte Ambulanzen und Praxen für Bronchiektasepatienten sein, die zum einen Patienten am Beginn zur Differenzialdiagnostik und zum Einschluss in Register- und Medikamentenstudien sehen und zum anderen Patienten mit komplexer oder rasch progredienter Erkrankung mitbetreuen.

\section{Inhalative Antibiotika überzeugten in aktuellen Studien nicht}

Kleinere und zum Teil bereits etwas ältere Studien konnten zeigen, dass Patienten mit chronischer Infektion durch Pseudomonas aeruginosa von einer inhalativen antimikrobiellen Suppressionstherapie hinsichtlich der Stabilisierung ihrer Lungenfunktion und Senkung der Exazerbationsrate profitieren können. Eine aktuelle Studie zu inhalativem Aztreonam konnte aber keine Überlegenheit im Vergleich zu Placebo nachweisen [6]. Diese Befunde stehen überraschenderweise im Gegensatz zu den

\footnotetext{
Das deutsche Bronchiektasen-Register PROGNOSIS

Um mehr in die Tiefe gehende Informationen über Bronchiektasen zu erhalten und um Prognosefaktoren und Therapieoptionen in Abhängigkeit

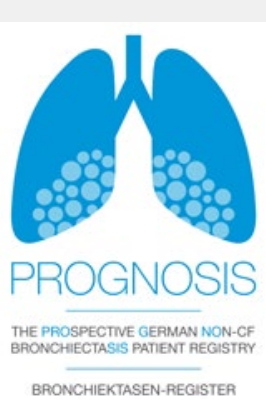
zur Ätiologie in Zukunft besser beurteilen zu können, wurde in den letzten Jahren ein nationales Register für diesen heterogenen Erkrankungskomplex aufgebaut (PROGNOSIS, Homepage: www.bronchiektasen-register.de). Es hat die Erstellung einer deutschsprachigen Leitlinie zur Diagnostik und zum Management von Non-CF-Bronchiektasen als eines seiner Ziele definiert.

Bei den Gründungszentren sind auch viele pneumologische Praxen vertreten, um die Versorgungsrealität besser abzubilden. Seit Juni 2015 werden Patienten rekrutiert. Erste Ergebnisse sind Mitte 2016 zu erwarten. Wie hilfreich diese für die Praxis sind, bleibt abzuwarten. Insgesamt positiv zu bewerten, ist sicherlich das zunehmende Interesse an einer zuletzt fast vergessenen Erkrankung.
}

Ergebnissen ähnlicher Untersuchungen bei Mukoviszidose, bei der dieses Antibiotikum aufgrund guter Wirksamkeit zugelassen ist. Auch inhalatives Colistin konnte in aktuellen Untersuchung bei Bronchiektasen mit einer chronischen Infektion durch Pseudomonas aeruginosa nicht überzeugen [7]. Ursächlich für die mangelnde Wirksamkeit dieser geprüften Substanzen scheinen Probleme im Studiendesign zu sein. Aus klinischer Erfahrung kann von einem deutlichen Nutzen inhalativer Antibiotika gesprochen werden, was aber noch in klinischen Studien bewiesen werden muss.

\section{Makrolide für alle?}

Unter der Dauertherapie mit Makrolid-Antibiotika (Azithromycin und Erythromycin) konnte die Exazerbationsrate in drei relativ großen Studien bei Bronchiektasen-Patienten signifikant gesenkt werden $[8,9,10]$. Dennoch wird ausdrücklich nicht empfohlen, bei jedem mit Bronchiektasen eine Therapie mit Makroliden zu beginnen. Empfohlen wird sie bei schwer erkrankten Patienten mit gehäuften Exazerbationen (mindestens oder mehr als zwei pro Jahr), chronischen Infektionen durch pathogene gramnegative Erreger und bei progredientem Lungenfunktionsverlust. Gastrointestinale und kardiale Nebenwirkungen sowie die Zunahme von Resistenzen wie bei Pneumokokken oder nicht-tuberkulösen Mykobakterien) können mit einer restriktiven Verordnung reduziert werden.

Literatur

1. Rademacher J, Ringshausen FC. Non-CF-Bronchiektasen mit Fokus auf die allergische bronchopulmonale Aspergillose. Pneumologie. 2013;67(1):40-47; quiz 48-9.

2. Li AM, Sonnappa S, Lex c et al. Non-Cystic fibrosis bronchiectasis: does knowing the aetiology lead to changes in management? Eur Resp J 2005; 26: 8-14.

3. Rademacher J, Ringshausen FC. Prevention and treatment of exacerbations of non-cystic fibrosis bronchiectasis. Eur Respir Monogr 2013; 60: 127-36.

4. Shoemark A, Ozerovitch L, Wilson R. . Aetiology in adult patients with bronchiectasis. Respir Med. Respir Med. 2007;101(6):1163-70.

5. Ringshausen FC, de Roux A, Diel R et al. Bronchiectasis in Germany: a population-based estimation of disease prevalence. Eur Respir J. 2015 Aug 20.

6. Barker AF, O'Donnell AE, Flume P et al. Aztreonam for inhalation solution in patients with non-cystic fibrosis bronchiectasis (AIR-BX1 and AIR$B X 2)$ : two randomised double-blind, placebo-controlled phase 3 trials. Lancet Respir Med. 2014;2(9):738-49.

7. Haworth CS, Foweraker JE, Wilkinson et al. Inhaled Colistin in Patients with Bronchiectasis and Chronic Pseudomonas aeruginosa Infection. Am J Respir Crit Care Med. 2014;189(8):975-82.

8. Altenburg J, de Graaff CS, Stienstra Y et al. Effect of azithromycin maintenance treatment on infectious exacerbations among patients with non-cystic fibrosis bronchiectasis: the BAT randomized controlled trial. JAMA. 2013;309(12):1251-9.

9. Serisier DJ, Martin ML, McGuckin MA, et al. Effect of long-term, low-dose erythromycin on pulmonary exacerbations among patients with non-cystic fibrosis bronchiectasis: the BLESS randomized controlled trial. JAMA. 2013;309(12):1260-7.

10. Wong C, Jayaram L, Karalus N, et al. Azithromycin for prevention of exacerbations in non-cystic fibrosis bronchiectasis (EMBRACE): a randomised, double-blind, placebo-controlled trial. Lancet. 2012:380(9842):660-7. 